\title{
Consideration of digital technology in dental implant treatment
}

\author{
WooHyung Jang, Sang-Won Park, Hyun-Pil Lim, Kwi-Dug Yun, Chan Park \\ Department of Prosthodontics, School of Dentistry, Chonnam National University, Gwangju, Korea
}

\begin{abstract}
Various considerations must be made when planning implant treatments using digital technology. These considerations include total integrated data, implant surgical guide CAD design, and an understanding of 3D printing technology. This paper analyzes these considerations to confirm more accurate and predictable digital technology. (JOURNAL OF DENTAL IMPLANT RESEARCH 2021;40(1):10-12)
\end{abstract}

Key Words: Digital technology, Dental implant, 3D printing, Total data, Dental CAD

\section{INTRODUCTION}

Currently, dental clinics are in a digital age ${ }^{1-3)}$. The grafting of digital technology to existing analog dental clinical technology is revolutionizing dental treatment. 'Digital dentistry', which is faster and with better quality than conventional procedures, brings great satisfaction to both patients and doctors. Among the various treatments in this field, the most one is "dental implant" treatment, which occupies the largest proportion of clinical dentistry. The introduction of digital technology for all diagnostics/surgical/prosthetic processes is currently at the core of successful implant treatment, and, in this regard, numerous academic studies are being reported and commercial technologies and products are being developed ${ }^{4,5}$.

As with all treatments, implant surgery, which is the first step, is the most important crucial part of establishing a treatment plan. However, there are many challenges in the application of digital technology in establishing a treatment plan in actual clinical practice. This is because, although a dental implant treatment is being considered, which includes the overall resources of a dental clinic, many clinicians are only accessing pieces of knowledge and information, rather than utilizing all available information. Numerous considerations are required to realize practical and efficient digital technology. It is difficult to use digital technology in clinical practice unless a complete workflow is established that includes accurate knowledge of $\mathrm{CBCT}$ for diagnosis, use of dental CAD software, and CAM or 3D printing for prosthesis fabrication ${ }^{6}$. Therefore, in this paper, we examine digital workflow for implant treatment as a whole and will specifically cover the considerations in the diagnosis and treatment planning stages.

\section{TECHNICAL NOTE}

\section{Dental implant treatment using digital technology}

Coachman et al. integrated digital application techniques for implant surgery for complete mouth implant rehabilitation ${ }^{7}$. This comprised the entire process including facial photo, panoramic radiographic, $\mathrm{CT}$, intraoral scanning, dental CAD software, and 3D printing technology. This paper showed that it is possible to perform implant treatment in one day using digital technology. Since then, many clinicians and dental companies have developed various digital technologies. The digital technology currently used for implant treatment is largely based on the

Received February 9, 2021, Accepted February 27, 2021.

(c) Journal of Dental Implant Research.

(i) $\$$ This is an open access article distributed under the terms of the Creative Commons Attribution Non-Commercial License (http://creativecommons.org/licenses/by-nc/4.0) which permits unrestricted non-commercial use, distribution, and reproduction in any medium, provided the original work is properly cited.

Correspondence to: Chan Park, Department of Prosthodontics, School of Dentistry, Chonnam National University, 33 Yongbong-ro, Buk-gu, Gwangju 61186, Korea. Tel: +82-62-530-5896, Fax: +82-62-530-5639, E-mail: upgradepc@jnu.ac.kr 


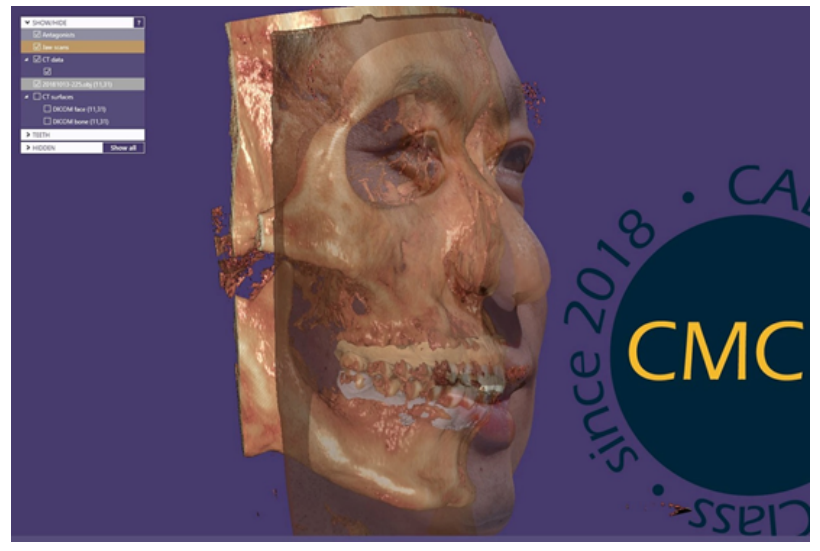

Fig. 1. Example of integrated data (CBCT, facial photo, intraoral scanning data).

acquisition of intraoral information through an oral scanner, the acquisition of extraoral information through a facial scanner, the acquisition of 3D data using CBCT, and the collection of integrated information. Based on this, diagnostic analysis is performed and various treatment plans are established through dental CAD. Then, a temporary/final prosthesis is produced and completed through CAM or 3D printing. These protocols have gradually evolved to enable accurate and rapid dental treatments.

\section{Importance of total data integration for implant guided surgery}

Data collection in the analog era was only one-dimensional according to each item. The oral environment was visually checked, photographs were taken, X-ray scans were obtained, and plaster models were made and set to the articulator. However, all data could not be combined into one entity and were integrated according to the clinician's own judgment.

Advances in digital devices have made it possible to accurately integrate these data. As suggested by Lee et al. ${ }^{8)}$, if CBCT is used as key data and facial scan data and intraoral scan data are combined, a diagnosis can be made through accurate patient data (Fig. 1). In addition, such data can be converted into big data in the future and applied to various AI technologies. The three-dimensional patient data are the most important factor necessary for accurate diagnosis, and thus the process of integrating these data is very important. In particular, as described above, CBCT, which is the basis of all data, is the most important and

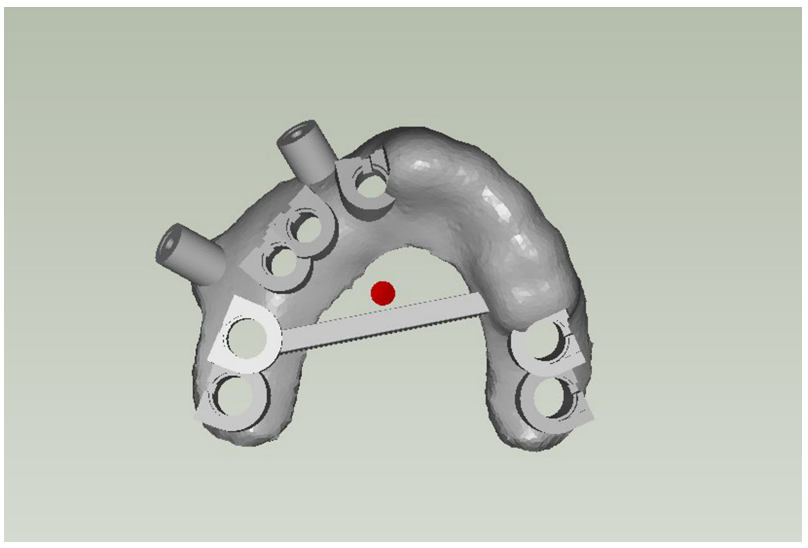

Fig. 2. Bar structure design for full-mouth surgical guide.

clinicians need to further confirm the accuracy and importance of CBCT for such integrated data collection.

\section{Points to note when designing a surgical guide for digital implant guide surgery}

The digital implant treatment proceeds in the order of diagnosis using the integrated data and the design and manufacture of an implant surgical guide through a digital dental CAD program ${ }^{4}$. Digital-based surgical stents provide the necessary guide for optimal implant placement. However, this assumes that the surgical guide has been properly manufactured. Therefore, the fabrication of the surgical guide must consider the design for accurate fabrication, along with the selection of the implant location according to the accurate diagnosis.

First, the design should be planned assuming the final prosthesis is predicted by an accurate diagnosis. This is also a fundamental reason for using digital methods. Second, if 3D printing is used to fabricate a full-mouth surgical guide, the amount of contraction in the posterior region must be considered. This is a limitation of 3D printing. This shrinkage can be addressed by setting the bar structure of both posterior teeth regions (Fig. 2). Third, it is necessary to consider a design to solve the watering problem (e.g. bone heating), which occurs when using a surgical guide (Fig. 3). Finally, if the guide cannot be used due to the mouth opening limit, it must be converted to an open-type design (Fig. 4). These considerations will facilitate a smooth digital guided implant treatment. 


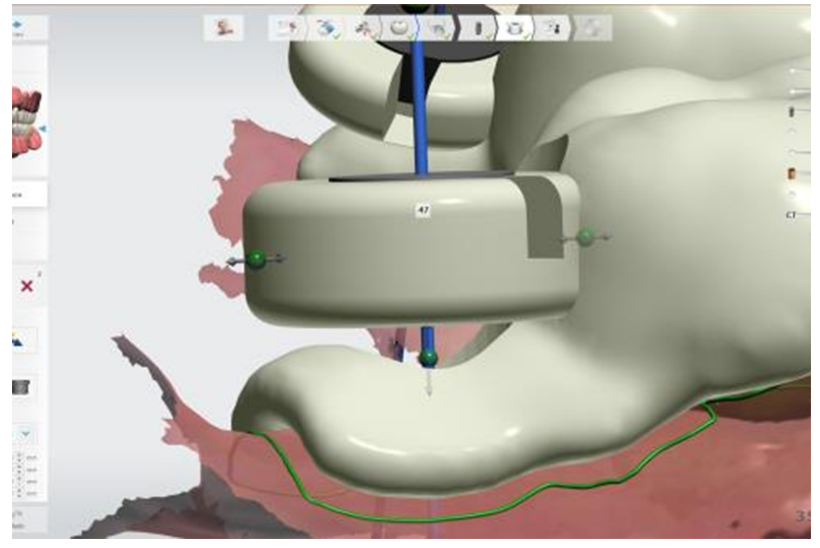

Fig. 3. Solution of watering problem by long guide sleeve.

\section{Understanding 3D printing required for surgical guide production}

Although there are several milling and 3D printing methods to manufacture digital surgical guide stents, economical and practical 3D printing methods are generally used $^{9}$. The photopolymerization method can be divided into stereolithography (SLA), digital light processing (DLP), and liquid crystal display (LCD ${ }^{10)}$. Thus, it is important to understand the advantages and disadvantages of each method. In addition, no 3D printing method is perfect and the shrinkage of photocurable polymers always exists. Therefore, it is necessary to be aware of these limitations ${ }^{11)}$. In addition, as 3D-printed products undergo a cleaning, supporter removal, and post-curing processes after printing, it is necessary to consider each process or understand how errors occurring in each process can be resolved.

\section{CONCLUSION}

The use of digital technology in implants has led to treatment processes becoming easier, more comfortable, and more accurate. However, for such a procedure to be possible, various factors must be considered. Accurately integrating all data, taking into account various factors when designing the guide, and overcoming limitations in manufacturing will bring satisfactory results for both the patient and operator.

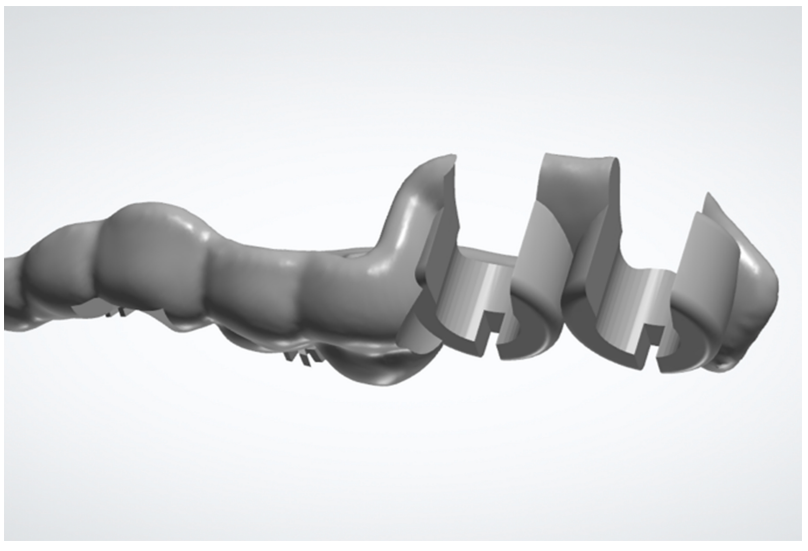

Fig. 4. Open sleeve design for patient with limited mouth opening.

\section{ORCID}

Chan Park, https://orcid.org/0000-0001-5729-5127

\section{REFERENCES}

1. Joda T, Ferrari M, Gallucci GO, Wittneben JG, Bragger U. Digital technology in fixed implant prosthdontics. Periodontal 2000 2017;73:178-92.

2. Takeuchi $\mathrm{Y}$, Koizumi H, Furuchi M, Sato Y, Ohkubo C, Matsumura $\mathrm{H}$. Use of digital impression systems with intraoral scanners for fabricating restorations and fixed dental prostheses. J Oral Sci 2018;60:1-7.

3. Millet C, Virard F, Dougnac-Galant T, Ducret M. CAD-CAM immediate to definitive complete denture transition : A digital dental technique. J Prosthet Dent 2020;124:642-6.

4. Greenberg AM. Digital technologies for dental implant treatment planning and guided surgery. Oral Maxillofac Surg Clin N Am 2015;27:319-40.

5. Patel N. Integrating three-dimensional digital technologies for comprehensive implant dentistry. J Am Dent Assoc 2010;141 Suppl 2:20S-4S.

6. Joda T, Ferrari M, Gallucci GO, Wittneben JG, Brägger U. Digital technology in fixed implant prosthodontics. Periodontol 2000 2017;73:178-92.

7. Coachman C, Calamita MA, Coachman FG, Coachman RG, Sesma N. Facially generated and cephalometric guided 3D digital design for complete mouth implant rehabilitation: a clinical report. J Prosthet Dent 2017;117:577-86.

8. Lee SY, Kim H, Lee D, Park C. Superimposition of a cone beam computed tomography (CBCT) scan and a photograph: A dental technique. J Prosthet Dent 2021;125:212-5.

9. Aimar A, Palermo A, Innocenti B. The role of 3D printing in medical applications: a state of the art. J Healthc Eng 2019; 5340616:1-10.

10. Quan H, Zhang T, Xu H, Luo S, Nie J, Zhu X. Photo-curing 3D printing technique and its challenges. Bioact Mater 2020;5: 110-5.

11. Stansbury JW, Idacavage MJ. 3D printing with polymers: Challenges among expanding options and opportunities. Dent Mater 2016;32:54-64. 